NASA/TM-1998-206298

$1 N \cdot 34$

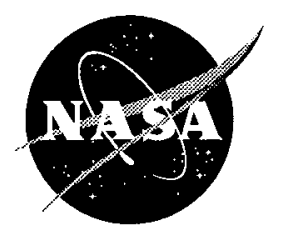

On the Environmental Realizability of Algebraically Growing Disturbances and Their Relation to Klebanoff Modes

Marvin E. Goldstein

Lewis Research Center, Cleveland, Ohio

David W. Wundrow

NYMA, Inc., Brook Park, Ohio 
Since its founding, NASA has been dedicated to the advancement of aeronautics and space science. The NASA Scientific and Technical Information (STI) Program Office plays a key part in helping NASA maintain this important role.

The NASA STI Program Office is operated by Langley Research Center, the Lead Center for NASA's scientific and technical information. The NASA STI Program Office provides access to the NASA STI Database, the largest collection of aeronautical and space science STI in the world. The Program Office is also NASA's institutional mechanism for disseminating the results of its research and development activities. These results are published by NASA in the NASA STI Report Series, which includes the following report types:

- TECHNICAL PUBLICATION. Reports of completed research or a major significant phase of research that present the results of NASA programs and include extensive data or theoretical analysis. Includes compilations of significant scientific and technical data and information deemed to be of continuing reference value. NASA's counterpart of peerreviewed formal professional papers but has less stringent limitations on manuscript length and extent of graphic presentations.

- TECHNICAL MEMORANDUM. Scientific and technical findings that are preliminary or of specialized interest, e.g., quick release reports, working papers, and bibliographies that contain minimal annotation. Does not contain extensive analysis.

- CONTRACTOR REPORT. Scientific and technical findings by NASA-sponsored contractors and grantees.
- CONFERENCE PUBLICATION. Collected papers from scientific and technical conferences, symposia, seminars, or other meetings sponsored or cosponsored by NASA.

- SPECIAL PUBLICATION. Scientific, technical, or historical information from NASA programs, projects, and missions, often concerned with subjects having substantial public interest.

- TECHNICAL TRANSLATION. Englishlanguage translations of foreign scientific and technical material pertinent to NASA's mission.

Specialized services that complement the STI Program Office's diverse offerings include creating custom thesauri, building customized data bases, organizing and publishing research results ... even providing videos.

For more information about the NASA STI Program Office, see the following:

- Access the NASA STI Program Home Page at http://www.sti.nasa.gov

- E-mail your question via the Internet to help@sti.nasa.gov

- Fax your question to the NASA Access Help Desk at (301) 621-0134

- Telephone the NASA Access Help Desk at (301) 621-0390

- Write to: NASA Access Help Desk NASA Center for AeroSpace Information 800 Elkridge Landing Road Linthicum Heights, MD 21090-2934 
NASA/TM-1998-206298

\section{On the Environmental Realizability of Algebraically Growing Disturbances and Their Relation to Klebanoff Modes}

Marvin E. Goldstein

Lewis Research Center, Cleveland, Ohio

David W. Wundrow

NYMA, Inc., Brook Park, Ohio

Prepared for the

International Symposium Theoretical and Computational Fluid Dynamics sponsored by the Florida State University

Tallahassee, Florida, November 6-8, 1996

National Aeronautics and

Space Administration

Lewis Research Center 


\section{Acknowledgments}

The authors would like to thank Dr. James Kendall for his helpful suggestions and sharing of data and

Professor Eli Reshotko for his encouragement.

Available from

NASA Center for Aerospace Information 800 Elkridge Landing Road

Linthicum Heights, MD 21090-2934

Price Code: A03
National Technical Information Service 5287 Port Royal Road Springfield, VA 22100 Price Code: A03 


\title{
ON THE ENVIRONMENTAL REALIZABILITY OF ALGEBRAICALLY GROWING DISTURBANCES AND THEIR RELATION TO KLEBANOFF MODES
}

\author{
M. E. Goldstein \\ National Aeronautics and Space Administration \\ Lewis Research Center \\ Cleveland, Ohio 44135 \\ Telephone: (216) 433-5825 \\ Fax: (216) 433-5266 \\ and \\ David W. Wundrow \\ NYMA, Inc. \\ Brook Park, Ohio 44142
}

\begin{abstract}
A theoretical explanation of some experimentally observed phenomena associated with the so-called Klebanoff modes is obtained by analyzing the flow over a finite thickness flat plate resulting from a small-amplitude distortion imposed on the upstream mean flow. The analysis shows (among other things) how the stretching of the vortex lines around the plate leads to streamwise vorticity at the plate surface, which then produces a streamwise velocity perturbation within the boundary layer that can be related to the experimentally observed Klebanoff mode. The complete evolution of this flow must be found by solving the boundary-region equations of Kemp (1951) and Davis and Rubin (1980), but a limiting analytical solution can also be obtained. Since the initial growth of the boundary-layer disturbance is nearly algebraic, our results demonstrate how the algebraically growing disturbances promoted by Landahl and others can be generated by a realistic external-disturbance environment. The relationship between these results and various bypass transition mechanisms is discussed.
\end{abstract}

\section{INTRODUCTION}

There are a number of different environmental disturbances that can cause transition to turbulence in boundary layers - with acoustic disturbances (Goldstein, 1985) probably being the most potent if they happen to be present in the flow. Another type of environmental disturbance that has received considerable attention in the literature is freestream turbulence, which was actually studied by Dryden (1936), but the later investigations of Klebanoff (1971) seem to be more well known.

Klebanoff measured the velocity fluctuations in a laminar flat-plate boundary layer due to externally imposed turbulence in a free stream (Reshotko, 1994) with nominally uniform upstream velocity. Figure 1 is a plot made by Klebanoff that has been shown around quite a bit, but, to our knowledge, has not actually been published in a journal article. He found, among other things, that the streamwise velocity fluctuations $U_{\infty} u^{\prime}$ peak at an $\eta$ of about 2.3 and attain a maximum value of about one percent of the undisturbed free-stream velocity $U_{\infty}$ (where $\eta$ is the usual Blasius variable, i.e., the normal coordinate $\mathrm{n}$ divided by the boundary-layer thickness $\delta_{0}=\sqrt{v \mathrm{x} * / \mathrm{U}_{\infty}}$ with $\mathrm{x} *$ denoting the distance along the plate, and $v$ denoting the kinematic viscosity). However, Klebanoff's most important observation was that the measured hot-wire signal passed through a low-pass filter at $12 \mathrm{~Hz}$ was almost identical in magnitude to the signal measured over all frequencies, indicating that most of the energy was at frequencies below $12 \mathrm{~Hz}$ (Reshotko, 1994). This result has now been reproduced many times by Leventhal and Reshotko (1981), Kendall (1985 and 1990), Arnal and Juillen (1978), Westin et al. (1994), and others. Kendall's lateral correlation studies show these structures to be very narrow in the spanwise direction-of the order of twice the displacement thickness $\delta^{*}$ in his particular case (where $\delta^{*} \approx 1.7 \delta_{0}$ ), while other experimenters find it to be nearly equal to $\delta^{*}$. Kendall called these low-frequency structures, Klebanoff modes, and that name seems to have stuck. 
Klebanoff (1971) proposed that these modes could be interpreted as a relatively small amplitude thickening and thinning of the Blasius boundary layer. Since perturbing the boundary-layer thickness $\delta_{0}$ in the Blasius solution $\mathrm{u}=\mathrm{F}_{\mathrm{B}}^{\prime}(\eta) ; \eta \equiv \mathrm{n} / \delta_{0}$ (where $\mathrm{F}_{\mathrm{B}}$ denotes the Blasius function, a prime denotes differentiation with respect to the argument, and all velocities are assumed to be normalized by $U_{\infty}$ ) by a small amount, say $\delta_{1}$, and expanding in a Taylor series gives

$$
u=F_{B}^{\prime}\left(n /\left(\delta_{0}+\delta_{1}\right)\right)=F_{B}^{\prime}(\eta)-\frac{\delta_{1}}{\delta_{0}} \eta F_{B}^{\prime \prime}(\eta)+\ldots
$$

This means that the difference between the actual streamwise velocity $u$ and the Blasius solution should be proportional to $\eta \mathrm{F}_{\mathrm{B}}$ ", which leads to the excellent agreement shown in figure 2-which is just a replot of the previous data.

This idea was presumably based on an earlier proposal by Bradshaw (1965) and put on a more or less rigorous analytical basis by Crow (1966) to explain the fairly large thickening and thinning of the boundary layer observed by Klebanoff and Tidstrom (1959) in their famous peak-valley splitting work, under presumably disturbance-free conditions where the mean flow would be expected to be two-dimensional. Bradshaw (1965), who observed similar behavior at the National Physical Laboratory, proposed that the observed thickening and thinning could be produced by a small spanwise-periodic variation in the upstream mean-flow direction.

Crow (1966) analyzed this phenomenon by considering a small-amplitude linearized sinusoidal perturbation of the flow over an infinitely thin flat plate, i.e., he considered the spanwise velocity perturbation (see fig. 3)

$$
\mathrm{w}_{\infty}=\varepsilon \cos 2 \pi \mathrm{z}
$$

imposed on a uniform flow $U_{\infty}$ over an infinitely thin plate, where $\varepsilon<<1$ is a measure of the disturbance amplitude, $\lambda$ is its characteristic spanwise length scale, and $\mathrm{z}=\mathrm{z}^{*} / \lambda$.

He assumed that the disturbance Reynolds number $\sigma \equiv R_{\lambda} \varepsilon$ (where $R_{\lambda} \equiv U_{\infty} \lambda / v$ ) is $0(1)$ and showed that the resulting streamwise boundary-layer velocity was of the form

$$
\mathrm{u}=\mathrm{F}_{\mathrm{B}}^{\prime}-\frac{1}{2}(\varepsilon \mathrm{x} \sin 2 \pi \mathrm{z}) \eta \mathrm{F}_{\mathrm{B}}^{\prime \prime}
$$

where $\mathrm{x}=\mathrm{x} * / \lambda$ is the scaled streamwise coordinate, which means that the corresponding boundary-layer thickness would be

$$
\delta=\delta_{0}\left(1+\frac{\varepsilon}{2} x \sin 2 \pi z\right)+\ldots
$$

\section{AN ALTERNATIVE PROPOSAL}

Crow's solution leads to a steady streamwise vorticity disturbance at the surface of the plate. On the other hand, the most general vortical distortion that can be imposed on the flow consists of all Fourier components of an arbitrary (unsteady) convected gust solution (Goldstein, 1978) of the linearized inviscid equations of motion. The streamwise vorticity component of the upstream motion was considered by Goldstein and Leib (1993a). However, here we look at another component of the upstream motion that might be visualized as the periodic steady wake flow shown in figure 4 , and can best be described as a spanwise variation in the streamwise velocity, so that the upstream vorticity is initially normal to the plate.

Our reason for considering this component is based on the following considerations. Firstly, the inviscid rapid distortion theory described below (see Hunt and Carruthers, 1990 and Goldstein, 1978, for additional details) shows that the inviscid vortex stretching around the plate tends to amplify the normal component of vorticity more than the other components. It also shows that, while the surface velocity remains bounded when the frequency of the disturbance is $0\left(U_{\infty} / \lambda\right)$, the zero-frequency (i.e., quasi-steady) component becomes logarithmically infinite at the surface of the plate-which means that the vortex stretching acts like a low-pass filter that preselects the low-frequency 
component of the motion. Secondly, the solution to the linearized unsteady boundary-layer equations (Guliaev et al., 1989) shows that the boundary layer on the surface of the plate also selectively amplifies the low-frequency portion of the spectrum with the small spanwise wavelength components undergoing the greatest amplification. The unsteady part of the flow actually moves out of the boundary layer at large values of $x$, (first into a kind of edge layer and then into the free stream) so that the boundary layer also acts as a low-pass filter for the free-stream turbulence.

These various mechanisms are responsible for producing the low-frequency elongated structures in the boundary layer which we have referred to as Klebanoff modes. Since the external turbulent flow behaves more or less linearly to lowest order of approximation, we can probably gain some useful insight into the dominant motion in the boundary layer by considering the most amplified Fourier component of that flow, which leads to the upstream boundary conditions described above.

While the inviscid vortex stretching around the plate tends to preselect the zero-frequency (i.e., $\omega<<U_{\infty} / \lambda$ ) component of the free-stream distortion, the boundary-layer flow itself actually selects the low-frequency components corresponding to $\omega \mathrm{x}^{*} / \mathrm{U}_{\infty}=0(1)$, where $\omega$ denotes the frequency, so that, while the external flow is quasisteady, the boundary-layer motion is actually governed by the unsteady boundary-layer equations (Guliaev et al., 1989). However, the purpose of this paper is to explain some of the relevant physical phenomena, rather than numerically reproduce the experimental results, and this can best be done by concentrating on the nonlinear phenomena and ignoring the unsteady effects.

Moreover, Watmuff (1997) found that he was able to reduce the peak RMS velocity in the Klebanoff modes by about 50 percent and make his measured contours of constant RMS velocity much more uniform by reordering his screens based on laser scans of the uniformity of the mesh. He was also able to directly measure weak spanwise nonuniformities in the mean flow velocity upstream of the plate which had an elongated streakiness in the transverse direction. Watmuff concludes from this that, "The evidence is almost overwhelming that the nonuniformities in the free stream (and hence the Klebanoff modes in the boundary layer) originate from small nonuniformities in the porosity of the wind tunnel screens." The wake-like distortion described above clearly provides an appropriate mathematical representation for this type of nonuniformity.

The stretching of the vortex lines around the plate not only amplifies the low-frequency component of the vorticity, but also produces the required streamwise vorticity at the plate surface as shown in figure 4 . This streamwise vorticity then leads to a perturbation to the streamwise boundary layer velocity that is similar to the result obtained by Crow. Both these velocity perturbations increase more or less linearly with the streamwise distance $\mathrm{x}^{*}$, and the solutions are therefore not uniformly valid in the streamwise direction, i.e., the perturbation velocity will become of the same order as the base flow when $\varepsilon \mathrm{x}=0(1)$ (see (3)). Moreover, the continued downstream growth of the mean boundary layer eventually causes the boundary-layer thickness to become of the same order as the spanwise wave length of the upstream distortion.

These linearized boundary-layer analyses can therefore only predict the initial stages of the boundary-layer development, and certainly not the inherently nonlinear processes that occur further downstream which may, as we shall see, be important for predicting the ultimate transition to turbulence. Moreover, the experiments show (as noted above) that the spanwise wave length of the dominant motion in the boundary layer (i.e., the Klebanoff mode) is of the same order as the boundary-layer thickness in the region where the measurements are carried out.

\section{ASYMPTOTIC STRUCTURE OF FLOW}

This leads us to consider the flow configuration depicted in figure 5. As in the Crow (1966) analysis, we allow the disturbance Reynolds number $\sigma$ to be $0(1)$, but now suppose that there is a wake-type velocity distortion imposed on the flow at a distance $0(\lambda / \varepsilon)$ upstream of the leading edge, where, as before, $\varepsilon$ is the amplitude of the distortion and $\lambda$ is its characteristic spatial scale. We also suppose that the plate thickness is $O(\lambda)$. Then in the asymptotic limit $\varepsilon \rightarrow 0, R_{\lambda} \rightarrow \infty$, with $\sigma=\varepsilon R_{\lambda}$ held fixed, the flow divides itself into four distinct asymptotic regions. The first (Region (1) in fig. 5) is a primarily inviscid region of dimensions $O(\lambda)$ surrounding the leading edge where the motion is a linear perturbation about a steady potential flow-which is the fundamental assumption for a branch of fluid mechanics now commonly referred to as linear-rapid-distortion theory (Hunt and Carruthers, 1990). Classical rapid distortion theory (RDT) assumes that the disturbance length scale, $\lambda$ is much smaller than the mean-flow length scale. Here we follow the modern approach and allow these length scales to be of the same order. This theory has its origins in a marvelous paper by Sir James that he called simply "Drift" and published in the first issue of the Journal of Fluid Mechanics (1956). Lighthill considered the flow around an infinite circular cylinder and assumed that the imposed upstream distortion varied linearly with $z$, rather than being periodic as in the present study. 
Below Region (1) (i.e., the RDT region) is a region (Region (2) in fig. 5) where the flow is governed by the linearized laminar boundary-layer equations-as it is in the Crow (1966) analysis-with the resulting solution being somewhat similar to the one found by Crow-at least to the lowest order of approximation. As in Crow's analysis, the linear boundary-layer solution breaks down when $\varepsilon x$ becomes large, and a new solution has to be obtained when $\overline{\mathrm{X}}=\varepsilon \mathrm{X}$ is order one, i.e., in Region (3). The flow in Region (3) is now fully three-dimensional, even though the crossflow velocity $w$ is still small, i.e., $0(\varepsilon)$. This is because the streamwise length scale is now large enough to allow a balance between the streamwise and spanwise convection terms within the governing equations. The flow in this region is then governed by what were originally referred to as the "boundary-region equations" by Kemp (1951), who was the first to use them, but that terminology seems to have gotten lost over the years. In any event, they are simply the Navier-Stokes equations with streamwise derivatives neglected in the viscous and pressure gradient terms and, more importantly, correspond to a rational asymptotic limit of the Navier-Stokes equations for the flows of the type being considered here. In the present paper, we resurrect the original terminology and refer to this approximate form of the Navier-Stokes equations as the boundary-region equations.

Finally, the flow in the large outer Region (4) is very simple and corresponds to a slow viscous decay of the imposed upstream velocity perturbation

$$
\mathbf{u}=1+\varepsilon \mathbf{u}_{\infty}(\mathbf{z}) \mathrm{e}^{-\overline{\mathbf{x}} / \sigma}+\ldots
$$

over the long streamwise length scale which is balanced by a weak crossflow velocity

$$
\mathrm{w}=\frac{\varepsilon}{\sigma} \frac{\partial \mathrm{u}}{\partial \mathrm{z}}
$$

needed to satisfy continuity. As in the Crow analysis, we have put $x=x * / \lambda, y=y^{*} / \lambda, z=z^{*} / \lambda$ and have assumed, for simplicity, that $u_{\infty}=a \cos 2 \pi \mathrm{z}$, where $\mathrm{a}=$ constant, i.e., that the upstream distortion is purely harmonic. The result can easily be generalized to an arbitrary periodic function of $z$, but the final result is not as transparent, and the physical phenomena can probably be better illustrated by considering only a single harmonic component.

The upstream boundary condition has to be imposed at a finite distance upstream of the leading edge, say L, on the long streamwise length scale $\bar{x}$, but this corresponds to upstream infinity on the rapid inner scale $x$ on which the potential flow field about the plate actually varies. So the upstream boundary condition is more or less unaffected by the presence of the plate in the strict asymptotic sense (but see Section 2.5 of Goldstein, 1978). Finally, the solutions in each of the individual Regions (1) to (4) must match together in the matched asymptotic expansion sense (Van Dyke, 1975).

\section{THE RAPID DISTORTION THEORY SOLUTION}

The flow in Region (1) (the RDT region) is given by the simple formula (Goldstein, 1978)

$$
\overrightarrow{\mathrm{u}}=\nabla \Phi+\varepsilon\left[\nabla \phi+\mathrm{au}_{\infty}(\mathrm{z}) \nabla \Delta\right]
$$

where $\Phi(\mathrm{x}, \mathrm{y})$ is the known potential for the mean flow about the plate (including the mean boundary-layer displacement effects, if necessary), $a=e^{-L / \sigma}$ is a constant, $u_{\infty}(z)$ is still assumed to be purely harmonic, and $\Delta(x, y)$ is the famous Lighthill (1956)-Darwin (1953) drift function to which the title of Lighthill's (1956) paper refers. Its difference between any two points on a streamline is equal to the time it takes a fluid particle to traverse that distance. It can be expressed as a simple integral (Lighthill, 1956; Goldstein, 1978; Darwin, 1953). Finally, the "perturbation potential" $\phi(x, y, z)$ (which is produced by the interaction of the upstream distortion with the flow field of the finite thickness plate) is determined by the simple Poisson's equation

subject to the boundary conditions

$$
\nabla^{2} \phi=-\mathrm{au}_{\infty} \nabla^{2} \Delta
$$

$$
\phi \rightarrow 0 \quad \text { as } \quad \mathrm{x} \rightarrow-\infty
$$




$$
\frac{\partial \phi}{\partial \mathrm{n}}+\mathrm{au}_{\infty} \frac{\partial \Delta}{\partial \mathrm{n}}=0 \text { on surface of plate. }
$$

Its solution shows that the crossflow velocity w exhibits the well-known logarithmic singularity

$$
\mathrm{w} \rightarrow \frac{\mathrm{au}_{\infty}^{\prime}}{\alpha} \ln \Psi,
$$

as the mean-flow stream function $\Psi(x, y)$ goes to 0 at the surface of the plate (Lighthill, 1956; Goldstein, 1978) where $\alpha$ is a constant related to mean potential flow in the vicinity of the forward stagnation point. Since a similar analysis of the unsteady flow shows that the $0\left(\mathrm{U}_{\infty} / \lambda\right)$ frequency components of the motion remain bounded at the surface of the plate, this result justifies our observations (in Section 2 above) about the selective amplification of the low-frequency component of the motion.

The solution to the boundary-value problem (8) to (10) must, in general, be found numerically. However, we are mainly interested in the flow in the nonlinear Region (3), and the upstream boundary conditions for this region can be obtained from the asymptotic solution to the RDT problem as $\mathrm{x} \rightarrow \infty$. Fortunately, this result can be obtained independently of the upstream solutions (Goldstein and Durbin, 1980; Goldstein, Leib, and Cowley, 1992) by (analytically) solving a simple equation with constant coefficients. The resulting solution, which is the same as in Goldstein, Leib, and Cowley (1992), depends on the upstream history effects only through the asymptotic drift function $\Delta_{+}+\mathrm{x}$, where $\Delta_{+}$is a suitable integral over the particle paths from upstream infinity to downstream infinity. The latter exhibits the logarithmic singularity

$$
\Delta_{+} \rightarrow \Delta_{0}(\mathrm{n})-\frac{1}{\alpha} \ln \mathrm{n} \text { as } \mathrm{n} \rightarrow 0
$$

at the surface of the plate corresponding to the log singularity in (11).

\section{THE LINEAR BOUNDARY-LAYER SOLUTION}

Region (2) coincides with the boundary layer on the surface of the flat plate. The relatively mild singularity in the inner limit of the Region (1) solution (11), which forms the outer limit of the Region (2) solution, can be smoothed out by viscous effects (Goldstein, Leib, and Cowley, 1992; Toomre, 1960) in Region (2). In fact, the mean boundary layer is again of the Blasius type sufficiently far downstream in the flow, and an analysis similar to that of Crow (1996) shows that the distorted streamwise velocity is now given by

$$
\mathrm{u} \rightarrow \mathrm{F}_{\mathrm{B}}^{\prime}+\varepsilon \mathrm{x} \ell \mathrm{n}\left(\frac{\varepsilon \mathrm{x}}{\sigma}\right)^{\frac{1}{2}} \frac{\mathrm{au}_{\mathrm{o}}^{\prime \prime}(\mathrm{z})}{4 \alpha} \eta \mathrm{F}_{\mathrm{B}}^{\prime \prime}+O(\varepsilon \mathrm{x})
$$

to lowest order of approximation. This result differs from Crow's because the gauge function $\varepsilon x \ln (\varepsilon x)$ is larger than his by the factor $\ell \mathrm{n}(\varepsilon x)$ in order to accommodate the log singularity in the external flow. However, the next order (i.e., $0(\varepsilon)$ ) terms in (13) would be significantly different from those obtained by Crow (1966) (whose lowest order perturbation is $0(\varepsilon \mathrm{x})$ ).

\section{THE LARGE-DISTURBANCE-REYNOLDS-NUMBER LIMIT OF THE BOUNDARY-REGION EQUATION SOLUTIONS AND THE FORMATION OF TURBULENT SPOTS}

The asymptotic solution (13) provides the upstream matching condition (i.e., the upstream boundary condition) for the flow in the larger region downstream (Region (3) in fig. 5). While the solution in this region is parabolic and can therefore be found by downstream marching, it still constitutes a relatively difficult numerical problem (Davis and Rubin, 1980; Wundrow, 1996). It therefore seems desirable to obtain some simplified analytical results. This 
can be done by considering the large- and small-disturbance-Reynolds-number limits. In the small-disturbanceReynolds-number limit, the boundary-region equations become linear. Here we discuss only the large-disturbanceReynolds-number limit in which $\sigma \equiv \varepsilon \mathbf{R}_{\lambda}$ goes to infinity (as was done in Goldstein, Leib, and Cowley, 1992, and Goldstein and Leib, 1993a,b).

In the large- $\sigma$ limit, the downstream nonlinear flow splits into two layers when $\overline{\mathrm{x}}_{1} \equiv \frac{\varepsilon \mathrm{x}}{\alpha} \ln (\lambda \bar{\delta})=0(1)$ where the new gauge function $\bar{\delta}(\sigma)$ is determined by $\frac{\lambda}{\bar{\delta}} \equiv \sqrt{\sigma \ell \mathrm{n}(\lambda \bar{\delta})}$ : a viscous wall layer in which the flow is governed by the three-dimensional, boundary-layer equations with no pressure-gradient terms-rather than by the boundaryregion equations and an outer inviscid vorticity layer induced by the log singularity in the upstream RDT solution. The scaled crossflow velocity $\mathrm{W}=\alpha \mathrm{w} / \varepsilon \ell \mathrm{n}(\lambda / \bar{\delta})$ in this latter region is determined by the inviscid Burgers equation (see fig. 6)

$$
\frac{\partial \mathrm{W}}{\partial \overline{\mathrm{x}}_{1}}+\mathrm{W} \frac{\partial \mathrm{W}}{\partial \mathrm{z}}=0
$$

which can, of course, be solved analytically. This solution can then be used to obtain the appropriate outer-edge boundary condition for the viscous wall layer whose thickness $\bar{\delta}$ is now smaller than $\lambda$ by a factor of the square root of $\sigma$ (the large disturbance Reynolds number) times $\ell \mathrm{n} \lambda \bar{\delta}$ (Goldstein, Leib, and Cowley, 1992). The boundary condition is that the streamwise velocity $\mathrm{U}$ goes to one, and the scaled crossflow velocity $\mathrm{W}$ goes to

$$
\mathrm{W} \rightarrow \mathrm{f}\left(\mathrm{z}-\overline{\mathrm{x}}_{1} \mathrm{~W}\right) \text { as } \overline{\mathrm{y}} \equiv \mathrm{n} / \bar{\delta} \rightarrow \infty
$$

where

$$
f(\zeta)=-u_{\infty}^{\prime}(\zeta)
$$

$\mathrm{u}_{\infty} \mathrm{z}$ is the imposed upstream velocity perturbation discussed above, and the primes denote differentiation with respect to the total argument (Goldstein, Leib, and Cowley, 1992).

The solution to this boundary-value problem can be expressed in terms of the Blasius solution by

$$
\mathrm{U}=\mathrm{F}_{\mathrm{B}}^{\prime}(\bar{\eta})
$$

where $\bar{\eta}$ is now given by

$$
\bar{\eta}=\frac{\left(1+\overline{\mathrm{x}}_{1} \mathrm{f}^{\prime}\right) \overline{\mathrm{y}}}{\left\{\frac{1}{3 \mathrm{f}^{\prime}}\left[\left(1+\overline{\mathrm{x}}_{1} \mathrm{f}^{\prime}\right)^{3}-1\right]\right\}^{\frac{1}{2}}},
$$

rather than by the usual Blasius variable. Timoshin (1992) recently used a similar transform to solve a very different boundary-layer problem. Since $\bar{\eta}$ now depends on $\mathrm{z}, \mathrm{U}$ also exhibits this dependence, and the solution is therefore directly interpretable as a localized thickening and thinning of the Blasius boundary layer. It is a natural extension of the linearized Crow-type solution into the nonlinear region. In fact, the large- $\sigma$ limit of the linear boundary-layer solution (13) can be recovered by expanding $\mathrm{F}_{\mathrm{B}}$ and $\bar{\eta}$ in a Taylor series for small $\bar{x}_{1}$-so the solution (16) clearly matches onto the appropriate linear solution in Region (2) as $\bar{x}_{1} \rightarrow 0$.

Of course, the solution to Burgers equation (14) eventually develops a singularity at a finite downstream position and is discontinuous or multivalued downstream of that point. This introduces a line of singularities into the vorticity-layer solution and a surface of discontinuity downstream of that line (Goldstein, Leib, and Cowley, 1992). (See fig. 7.) A new inviscid solution, which accounts for streamline curvature effects, can then be constructed to eliminate the line singularity, and (downstream of that) an inviscid solution that brings in pressure-gradient effects can be constructed to eliminate the surface discontinuity (Goldstein and Leib, 1993b). This is all rather intricate, but 
the important thing to note is that this solution is still inviscid and has the general structure shown in figure 7

(Goldstein, Leib, and Cowley, 1992; Goldstein and Leib, 1993b). However, the downstream pressure-gradient solution imposes its strong pressure gradients on the underlying boundary layer and causes it to develop a singularity at a finite downstream position. This singularity is an indication that the boundary-layer approximation breaks down, and a new (less approximate) set of equations has to be used to describe the flow. Since the spanwise length scale decreases and the boundary-layer thickness increases in the vicinity of the singularity, this probably turns out to be the full boundary-region equations.

The boundary-layer velocity profile eventually becomes much flater and can even become inflectional in the viscinity of the singularity. (See Goldstein, Leib, and Cowley, 1992.) The flow therefore becomes susceptible to rapidly growing Tollmien-Schlichting waves and possibly even Rayleigh instabilities which could ultimately lead to the formation of turbulent spots. It therefore provides a possible "bypass" mechanism that could lead to transition without involving a more global Tollmien-Schlichting wave system (but see below).

Figure 8 is a plot (taken from Goldstein, Leib, and Cowley, 1992) of constant streamwise velocity contours in the crossflow plane calculated at various streamwise locations with $\mathrm{u}_{\infty}$ set equal to $\cos \mathrm{z}$. The initial spanwise variation in the linear regime is, of course, sinusoidal, and the subsequent effect of the nonlinearity is to produce a sharp focusing along the lines of maximal thickness, with the attendant introduction of progressively shorter streamwise length scales into the flow.

Since the large streamwise velocity perturbations are concentrated in narrow spanwise regions, the spanwise average RMS velocity

$$
\mathrm{u}^{\prime}\left(\overline{\mathrm{x}}_{1}, \eta\right)=\left\{\frac{1}{\pi} \int_{0}^{\pi}\left[\mathrm{U}\left(\overline{\mathrm{x}}_{1}, \eta, \mathrm{z}\right)-\mathrm{F}_{\mathrm{B}}^{\prime}(\eta)\right]^{2} \mathrm{dz}\right\}^{\frac{1}{2}}
$$

(which roughly corresponds to the local RMS velocity measurement in the real flow) will be much smaller than the maximum velocity fluctuation. This is shown in figure 9 , in which the solid curve is the peak value (relative to $\eta$ ) of the RMS velocity (18), while the dashed curve is the peak (with respect to $\eta$ ) of the maximum velocity fluctuation

$$
\left|\mathrm{U}\left(\overline{\mathrm{x}}_{1}, \eta, 0\right)-\mathrm{F}_{\mathrm{B}}^{\prime}(\eta)\right|
$$

with U determined by (16) and (17) in both cases. Notice that while the RMS velocity fluctuation is only a little over 10 percent at $\bar{x}_{1} \simeq 0.75$, the maximum velocity fluctuation is nearly equal to 50 percent at this point. Figure 8 shows that this is large enough to produce localized nonlinear behavior, while the straight-line behavior of the solid curve suggests that the RMS velocity continues to behave linearly.

The experiments (see comprehensive summary in table III of Westin, et al., 1994) show that the Klebanoff mode RMS velocity fluctuations can easily exceed 10 percent. The present results, therefore, suggest that the corresponding maximum velocity fluctuations will then be large enough to produce the localized breakdown of the boundary-layer solution alluded to above, even though the RMS velocity fluctuations are still relatively small. Needless to say, nonlinear theory will have to be used to calculate the flow.

Figure 10 is a flow visualization by Kendall (1985) using a smoke-wire technique to view the crossflow plane with a camera located $3 \mathrm{~m}$ downstream of the test section. The smoke wire was located at a Blasius $\eta$ value of about 1.5. This figure suggests that the crossflow distortion is focused in rather narrow spanwise regions in agreement with the theoretical predictions.

\section{APPLICATION OF SOLUTION TO KENDALL'S EXPERIMENT}

Figure 11 shows some data taken by Kendall $(1985,1990)$ in an experiment similar to Klebanoff's. The closed symbols show the agreement of the mean velocity distribution with the Blasius profile. The open symbols are the filtered signal at the Tollmien-Schlichting wave frequency. This disturbance is clearly small (note the 20 -fold magnification) and concentrated at the outer edge of the boundary layer-definitely not a T-S mode. The remaining curve represents the broad-band signal corresponding to the low-frequency Klebanoff mode. The solid curve is the $\eta \mathrm{F}_{\mathrm{B}}$ " mode shape proposed by Klebanoff and put on a more rigorous analytical basis by Crow (1966). Kendall's 
turbulence levels are higher than those of Klebanoff by a factor of about four or five, but the results are the same; the agreement with Crow's mode shape is excellent, except perhaps at the outer edge of the boundary layer where the data approaches a finite value while Crow's analytical distribution goes to zero. Figure 12 is a replot of the data in Figure 11, but the solid curve is now obtained from (18) with $U$ determined from (16) and (17). The agreement is now slightly better than it was with Crow's linear mode shape.

While Kendall did not observe any Tollmien-Schlichting waves in the region where the Klebanoff modes first become predominant, he did detect T-S wave packets further downstream. These wave packets frequently exhibited nonlinear amplitude dependence on the free-stream turbulence level, but much more interestingly, appeared to be highly intermittent-even though Kendall's measurements showed the free-stream turbulence to be relatively timestationary (Kendall, 1985).

A possible explanation for this, as well as for the nonlinear dependence on free-stream amplitude, is that the upstream Klebanoff modes act as receptivity sites for the free-stream turbulence (Goldstein, Leib, and Wundrow, 1992). At the low Mach numbers of Kendall's experiment, there is probably a significant mismatch between the T-S wave length and the wave length of the free-stream turbulence-with the latter being considerably longer than the former. It is therefore necessary that the boundary-layer flow contain some sort of short streamwise length scale that can "scatter" the long wavelength free-stream disturbance into the shorter T-S waves. It has been shown (Goldstein, 1983; Goldstein et al., 1983; Goldstein and Hultgren, 1989; Goldstein, 1985) that (1) imperfections in the plate surface, (2) the sudden change in the curvature at the junction between the leading-edge region and the flat portion of the plate, and (3) the rapid growth of the laminar boundary layer at the leading edge can all produce the requisite short streamwise length scale. Our proposal here is that the Klebanoff modes can also fulfill this role with the short streamwise length scales resulting from the previously shown focusing effect that occurs in the large- $\sigma$ asymptotic solution.

Since the Klebanoff modes are, in reality, not very steady, but rather meander across the plate at low frequencies, i.e., they are governed by the unsteady boundary-layer equations, the more or less localized receptivity centers would also be expected to move around and thereby cause the T.S. wave packets to appear to be intermittent to a downstream observer with a fixed probe.

\section{NUMERICAL SOLUTIONS FOR THE BOUNDARY-REGION EQUATIONS AND ALGEBRAIC DISTURBANCE GROWTH}

The appearance of logarithmic gauge functions in the large $\sigma$ asymptotic expansion for the solution in Region (3) (see Goldstein, Leib, and Cowley, 1992) suggests that this expansion is not very robust. The only recourse is to solve the full (or perhaps the linearized) boundary-region equations numerically. Here we discuss the full nonlinear case. The relevant initial conditions are determined by requiring that the solutions match onto the asymptotic solutions in the upstream RDT/linear boundary-layer region (Region (1) and (2) in Fig. 5). Since, as we have seen, the streamwise boundary-layer velocity grows nearly linearly with $x$ (actually it grows like $x \ln x$ ), the initial velocity in Region (3) will have to exhibit the same nearly algebraic growth. (This is, of course, also true for the large- $\sigma$ asymptotic solution described above, which actually exhibits purely linear growth to lowest order of approximation.)

This linear or nearly linear growth corresponds to the algebraic growth mechanism originally discussed for boundary layers by Hultgren and Gustavsson (1981), subsequently promoted by Landahl and his students (Landahl, 1990; Russell and Landahl, 1984) as a possible explanation for certain types of bypass transition, and now appears to be gaining increasing acceptance by the transition community as a viable alternative to instability wave mechanisms. We have applied it to spatially growing disturbances (in Goldstein, Leib, and Cowley, 1992, and Goldstein, Leib, and Wundrow, 1992) and, more importantly, shown how these latter disturbances can be generated by realistic disturbances in the environment (i.e., we have shown in Morkovin's words that these algebraically growing disturbances are "environmentally realizable"). Our results also suggest that it is probably necessary to account for nonparallel flow effects when dealing with disturbances that are both environmentally realizable and algebraically growing.

Figure 13 shows some preliminary solutions to the boundary-region equations taken from Wundrow (1996). These results roughly match onto the upstream RDT solution with $u_{\infty}$ still given by cos z. However, the initial condition has now been adjusted to make the velocity profiles more inflexional. The figure again shows contours of constant streamwise velocity in the crossflow plane at various streamwise locations. As expected, the initial flow is relatively two-dimensional with the perturbation varying sinusoidally in the z-direction. The figure also shows a 
fairly rapid departure from the initial two-dimensional state that becomes more nonharmonic with increasing downstream distance. The relative distortion becomes maximal somewhere downstream of $\xi=1.3$ (i.e., $x=0.3 \sigma / \varepsilon$ ) and then rapidly decays out with further increase in downstream distance due to the action of viscosity. So, unlike the large-disturbance-Reynolds-number limit that we discussed above, the boundary layer does not develope a singularity in this case.

The ideas discussed in this paper can be extended to the case where the upstream distortion is unsteady. The boundary layer will filter out most, but not all, of the unsteady components of the motion, and the flow in Region (3) will be described by the unsteady boundary-region equations. The relevant solution to these equations requires extensive numerical computation, which can, however, be greatly simplified by considering the small-disturbanceReynolds-number limit. This has been done in Leib, Wundrow, and Goldstein (1997).

\section{EFFECT OF KLEBANOFF MODES ON INSTABILITY WAVES}

At very low free-stream turbulence levels $(\sim 0.1 \%)$, even the Schubauer and Skramstad (1948) experiments show that the nonlinear breakdown of Tollmien-Schlichting waves are directly responsible for the appearance of turbulent spots. The situation is less clear at intermediate turbulence levels (of the order of 1 percent or so). Figure 13 of Westin et al. (1994) shows that there is enhanced streamwise amplification of the boundary-layer fluctuations within the band of frequencies corresponding to unstable Tollmien-Schlichting wave growth. However, these fluctuations tend to be broad band, rather than being concentrated in a narrow range about the most unstable frequency as in the low-turbulence-level experiments. But follow-on experiments by Boiko et al. (1994), in which discrete frequency Tollmien-Schlichting waves were artificially introduced into the flow, show that sufficiently high levels of free-stream turbulence (and, therefore, presumably the Klebanoff modes) can produce significant transfer of energy between frequencies within the unstable bands for Tollmien-Schlichting waves. It may therefore be possible that unstable Tollmien-Schlichting-type waves growing on a base flow computed from the unsteady boundary-region equations will exhibit a sufficiently broad band-width to explain the experimental observations. But in order to verify this, it is necessary to investigate the stability of this unsteady base flow, which is currently being done by the present authors. However, even without doing the calculation, it is clear that certain portions of an initially undistorted T.S. wave would grow faster than others as it propagates downstream, since certain portions of the base flow would be more unstable than others. In fact, the rapidly growing localized Tomlmien-Schlichting waves and/or Rayleigh instabilities that we discussed in conjunction with the large turbulent Reynolds-number, Klebanoff-mode solution could be part of a more global T.S. wave system that impinges on the locally destabilized region from upstream. This would certainly enhance the local growth of the T.S. wave over the two-dimensional case, even though the average growth could be much smaller than in the two-dimensional case.

The various mechanisms discussed in this paper could be operative in different flow regimes up to free-stream turbulence levels of about 5 percent. Beyond that point, it is impossible to detect any turbulent spots or, for that matter, any other discernible features of the flow.

\section{CONCLUDING REMARKS}

In all of the experiments of which the authors are aware, no matter what the free-stream turbulence level, the dominant streamwise velocity fluctuations are of the Klebanoff type, i.e., they are of very low frequency, have a peak value at a Blasius $\eta$-value of about 2.3 , are very elongated in the streamwise direction, and are very narrow in the spanwise direction. We have noted that this is consistent with the predictions of inviscid rapid-distortion theory, which shows that the vortex stretching around the plate selectively amplifies the low-frequency component of the motion. We also pointed out that it is consistent with the solutions to the linearized unsteady boundary-layer equations which, for the convected (i.e., pressureless) free-stream disturbances that describe the weak free-stream turbulence, grow linearly with the streamwise coordinate in the low-frequency limit and move out of the boundary layer (first into a kind of edge layer and then into the free stream) in the high-frequency limit; so that only low-frequency disturbances remain in the boundary layer at sufficiently large distances downstream.

Moreover, since it turns out that the streamwise velocity perturbations are driven by the spanwise gradient of the spanwise velocity, the streamwise velocity growth rate initially increases with decreasing spanwise length scale. This trend is eventually reversed by viscous effects in the downstream region where the boundary-layer thickness 
becomes of the same order as the spanwise length scale, and, in the most general case, the motion is governed by the unsteady boundary-region equations. But even if this selection did not occur, the free-stream turbulence and/or the mean-flow distortion which drive the Klebanoff modes would eventually run out of energy when the spanwise length scale became too short. These two factors combine to form a selection mechanism for the spanwise length scale, with one or the other becoming dominant, depending on the nature of the free-stream turbulence. This might explain the continuing argument among experimentalists as to whether the spanwise length scale is set by the freestream turbulence or by the boundary layer itself.

\section{ACKNOWLEDGMENT}

The authors would like to thank Dr. James Kendall for his helpful suggestions and sharing of data and Professor Eli Reshotko for his encouragement.

\section{REFERENCES}

Arnal, D. and Juillen, J.C. (June 1978). Contribution experimentale a l'etude de la receptivite d'une couche limite laminare, a la turbulence de l'ecoulement general. ONERA Rapport Technique No. 1/5018 AYD.

Boiko, A.V., Westin, K.J.A., Klingmann, B.G.B., Koslov, V.V. and Alfredsson, P.H. (1994). Experiments in a boundary layer subjected to free stream turbulence. Part 2 . The role of TS waves in the transition process. $J$. Fluid Mech. 281, pp. 219-245.

Bradshaw, P. (1965). The effect of wind-tunnel screens on nominally two-dimensional boundary layers. $J$. Fluid Mech. 22, part 4, pp. 679-687.

Crow, S.C. (1966) The spanwise perturbation of two-dimensional boundary layers. J. Fluid Mech. 24, pp. 153-164.

Darwin, C. (1953). A note on hydrodynamics. Proc. Camb. Phil. Soc. 49, pp. 342-354.

Davis, R.T. and Rubin, S.G. (1980). Non-Navier Stokes viscous flow computations. Comput. Fluids 8, p. 101.

Dryden, H.L. (1936). Air flow in the boundary layer near a plate. NACA Report 562.

Goldstein, M.E. (1978). Unsteady vortical and entropic distortions of potential flows round arbitrary obstacles. $J$. Fluid Mech. 89, pp. 433-468.

Goldstein, M.E. (1983). The evolution of Tollmien-Schlichting waves near a leading edge. J. Fluid Mech. 127, pp. 59-81.

Goldstein, M.E. (1985). Scattering of acoustic waves into Tollmien-Schlichting waves by small streamwise variations in surface geometry. J. Fluid Mech. 154, pp. 509-529.

Goldstein, M.E. and Durbin, P.A. (1980). The effect of finite turbulence spatial scale on the amplification of turbulence by a contracting stream. J. Fluid Mech. 98, part 3, pp. 473-508.

Goldstein, M.E. and Hultgren, L.S. (1989). Boundary layer receptivity to long-wave free-stream disturbances. Annual Review of Fluid Mechanics 21, pp. 137-166.

Goldstein, M.E., Leib, S.J. and Cowley, S.J. (1992). Distortion of a flat plate boundary layer by free stream vorticity normal to the plate. J. Fluid Mech. 237, pp. 231-260.

Goldstein, M.E., Leib, S.J. and Wundrow, D.W. (November 1992). Distortion of a flat plate boundary layer by freestream vorticity. Bulletin of the American Physical Society 37, No. 8, pp. 1778-1779.

Goldstein, M.E. and Leib, S.J. (1993a). Three-dimensional boundary-layer instability and separation induced by small-amplitude streamwise vorticity in the upstream flow. J. Fluid Mech. 246, pp. 21-41.

Goldstein, M.E. and Leib, S.J. (1993b). A note on the distortion of a flat-plate boundary layer by free steam vorticity normal to the plate. J. Fluid Mech. 248, pp. 531-541.

Goldstein, M.E., Sockol, P.M. and Sanz. J. (1983). The evolution of Tollmien-Schlichting waves near a leading edge. Part 2. Numerical determination of amplitudes. J. Fluid Mech. 129, pp. 443-453.

Guliaev, A.N., Kozlov, V.E., Kuznetson, V.R., Mineev, B.I. and Sekundov, A.N. (1989). Interaction of a laminar boundary layer with external turbulence. Izv. Akad. Nauk SSSR Mekh. Zhid. Gaza 6, pp. 700-710.

Hultgren, L.S. and Gustavsson, L.H. (1981). Algebraic growth of disturbances in a laminar boundary layer. Phys. Fluids 24, pp. 1000-1004.

Hunt, J.C.R. and Carruthers, D.J. (1990). Rapid distortion theory and the 'problems' of turbulence. J. Fluid Mech. 212, pp. 497-532. 
Kemp, N. (1951). The laminar three-dimensional boundary layer and a study of the flow past a side edge. M.Ae.S. Thesis, Cornell University.

Kendall, J.M. (July 1985). Experimental study of disturbances produced in a pre-transitional laminar boundary layer by weak free stream turbulence. AIAA Paper 85-1695.

Kendall, J.M. (1990). Boundary layer receptivity to free stream turbulence. AIAA Paper 90-1504.

Klebanoff, P.S. (1971). Effect of free-stream turbulence on a laminar boundary layer. Bulletin Am. Phys. Soc. 16.

Klebanoff, P.S. and Tidstrom, K.D. (1959). Evolution of amplified waves leading to transition. NASA TN-D-195.

Landahl, M.T. (1990). On sublayer streaks. J. Fluid Mech. 212, p. 243.

Leib, S.J., Wundrow, D.W. and Goldstein, M.E. (1997b). Effects of free-stream turbulence and other vortical disturbances on a laminar boundary layer. Submitted to the Journal of Fluid Mechanics.

Leventhal, L. and Reshotko, E. (1981). Preliminary experimental study of disturbances in a laminar boundary layer due to distributed surface roughness. AIAA Paper 81-1224.

Lighthill, M.J. (1956). Drift. J. Fluid Mech. 1, pp. 31-53.

Reshotko, E. (1994). Boundary layer instability, transition, and control. Dryden Lecture in Research, AIAA Paper 94-0001.

Russell, J.M. and Landahl, M.T. (1984). The evolution of a flat eddy near a wall in an inviscid shear flow. Phys. Fluids 27, pp. 557-570.

Schubauer, G.B. and Skramstad, H.K. (1948). Laminar boundary layer oscillations and transition on a flat plate. NACA Report 909.

Suder, K.L., O'Brien, J.E. and Reshotko, E. (1988). Experimental study of bypass transition in a boundary layer. NASA TM-100913.

Timoshin, S.N. (1992). Singularity in the solution of the equations of a three-dimensional boundary layer due to the collision of wall jets. Fluid Dynamics, Plenum Publishing Corp., pp. 538-543.

Toomre, A. (1960). The viscous secondary flow ahead of an infinite cylinder in a uniform parallel shear flow. $J$. Fluid Mech. 7, pp. 145-155.

Van Dyke, M.D. (1975). Perturbation Methods in Fluid Mechanics. Parabolic Press. Palo Alto, California

Watmuff, J.H. (1997). Detrimental effects of almost immeasurably small free-stream nonuniformities generated by wind tunnel screens. AIAA Paper 97-0228.

Westin, K.J.A., Boiko, A.V., Klingmann, B.G.B., Kozlov, V.V. and Alfredsson, P.H. (1994). Experiments in a boundary layer subjected to free stream turbulence. Part 1 . Boundary layer structure and receptivity. $J$. Fluid Mech. 281, pp. 193-218.

Wundrow, D.W. (1996). Linear instability of a uni-directional transversely sheared mean flow. NASA CR-198535. 


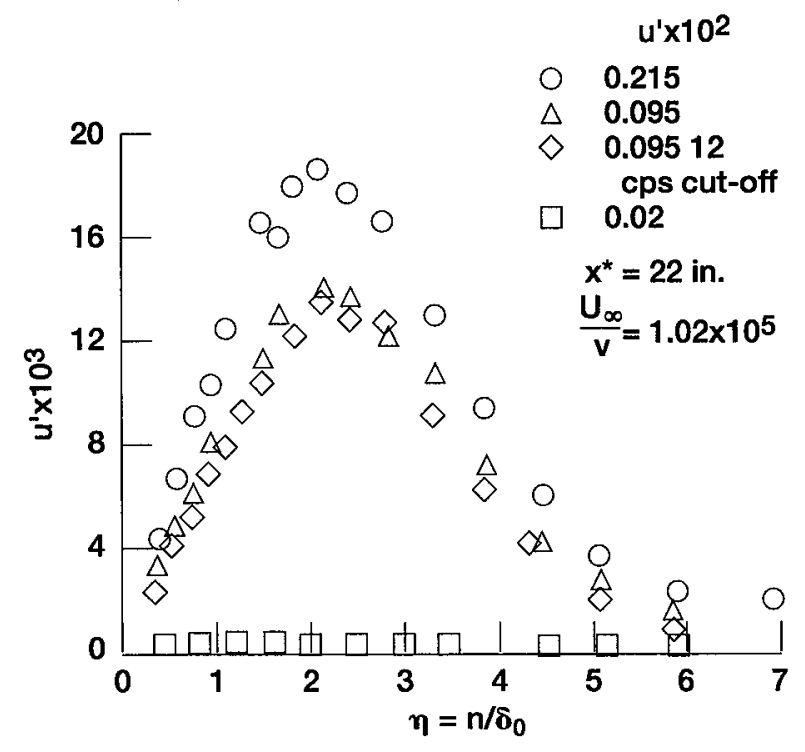

Figure 1.-Streamwise velocity fluctuations in laminar boundary layer measured by Klebanoff (unpublished data) $\eta=n / \delta_{0}$ where $n$ is normal distance to wall, $\delta_{0} \equiv \sqrt{x^{\star} v / U_{\infty}}$ is the Blasius boundary layer thickness.

The streamwise distance measured from leading edge $x^{\star}$ is equal to 22 inches.

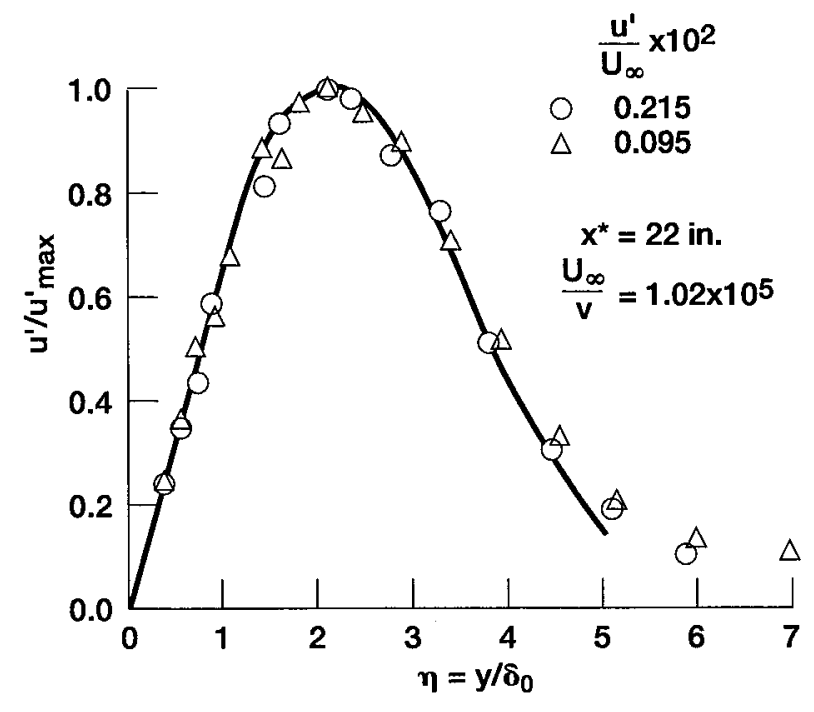

Figure 2.-Comparison of Klebanoff's data with Taylor series expansion of Blasius solution. Same data as in Figure 1.

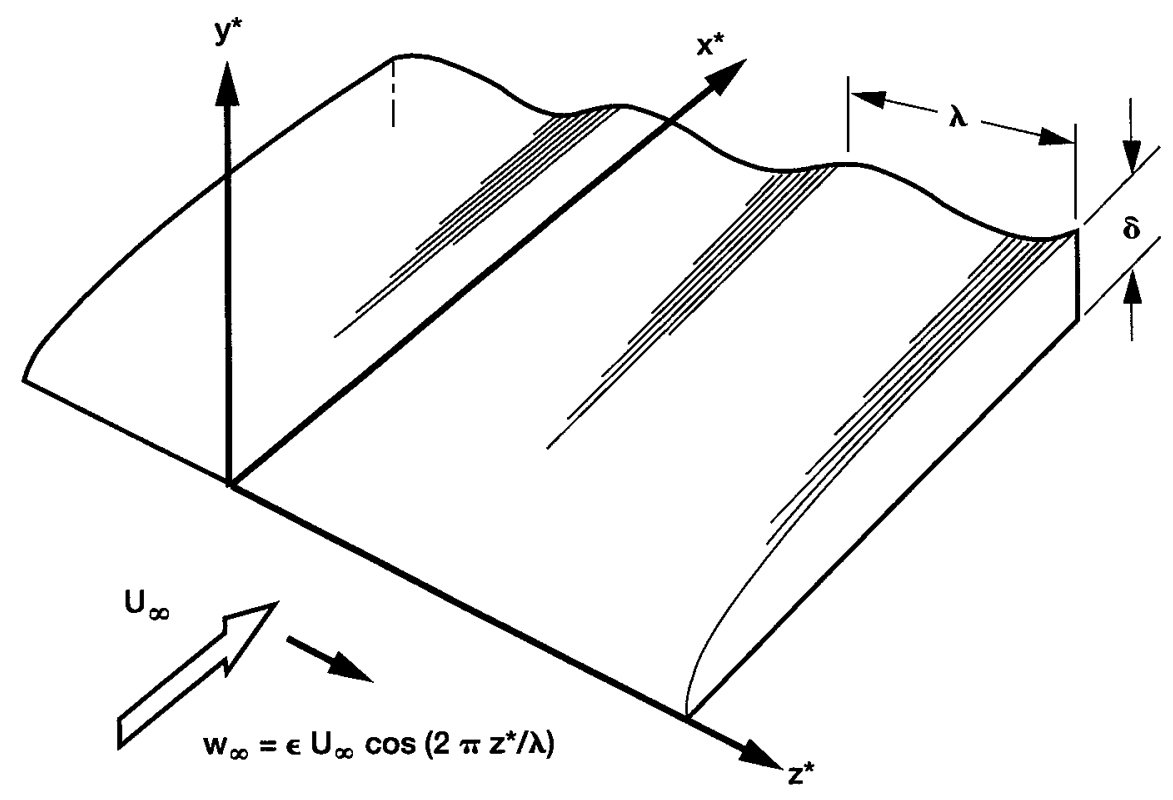

Figure 3.-Flow configuration analyzed by Crow (1996): $\lambda=$ spanwise wave length of distortion; $w_{\infty}=$ imposed crossflow velocity. 


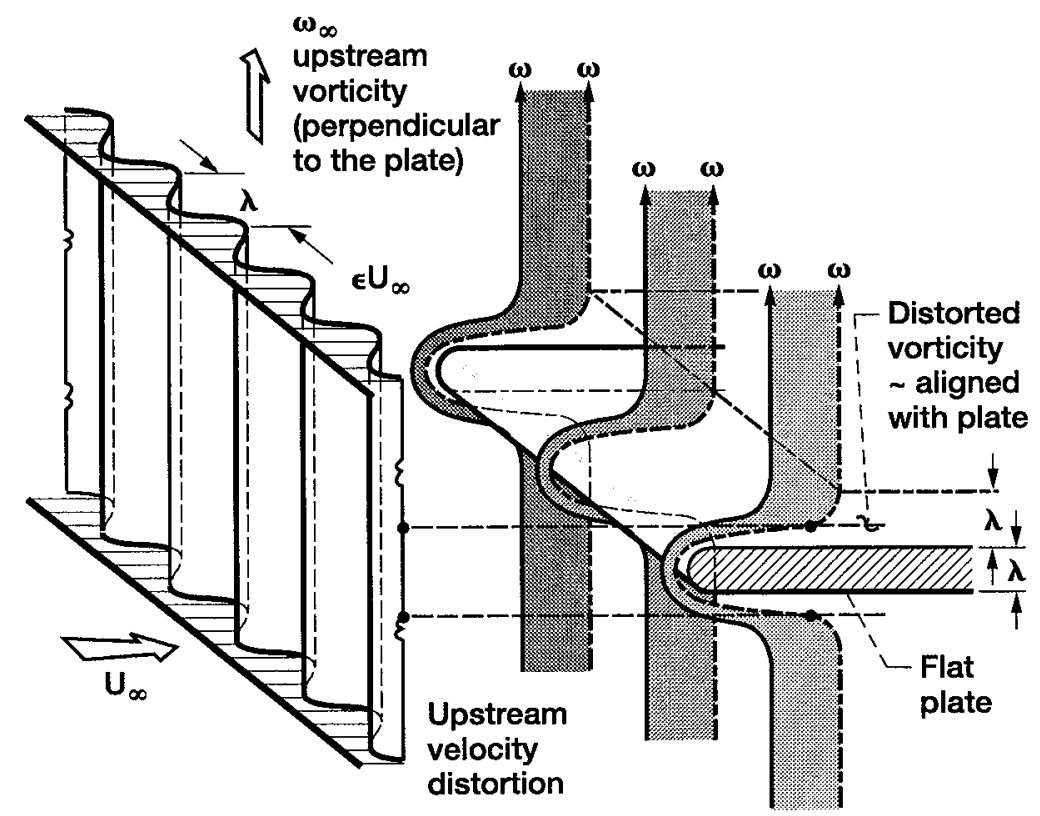

Figure 4.-Schematic view of stretching of vortex lines around plate: $\lambda=$ spanwise length scale of distortion, $\epsilon=$ characteristic scale of imposed streamwise velocity distortion, $\omega_{\infty}=$ imposed vorticity distortion, $\omega=$ local vorticity lines.

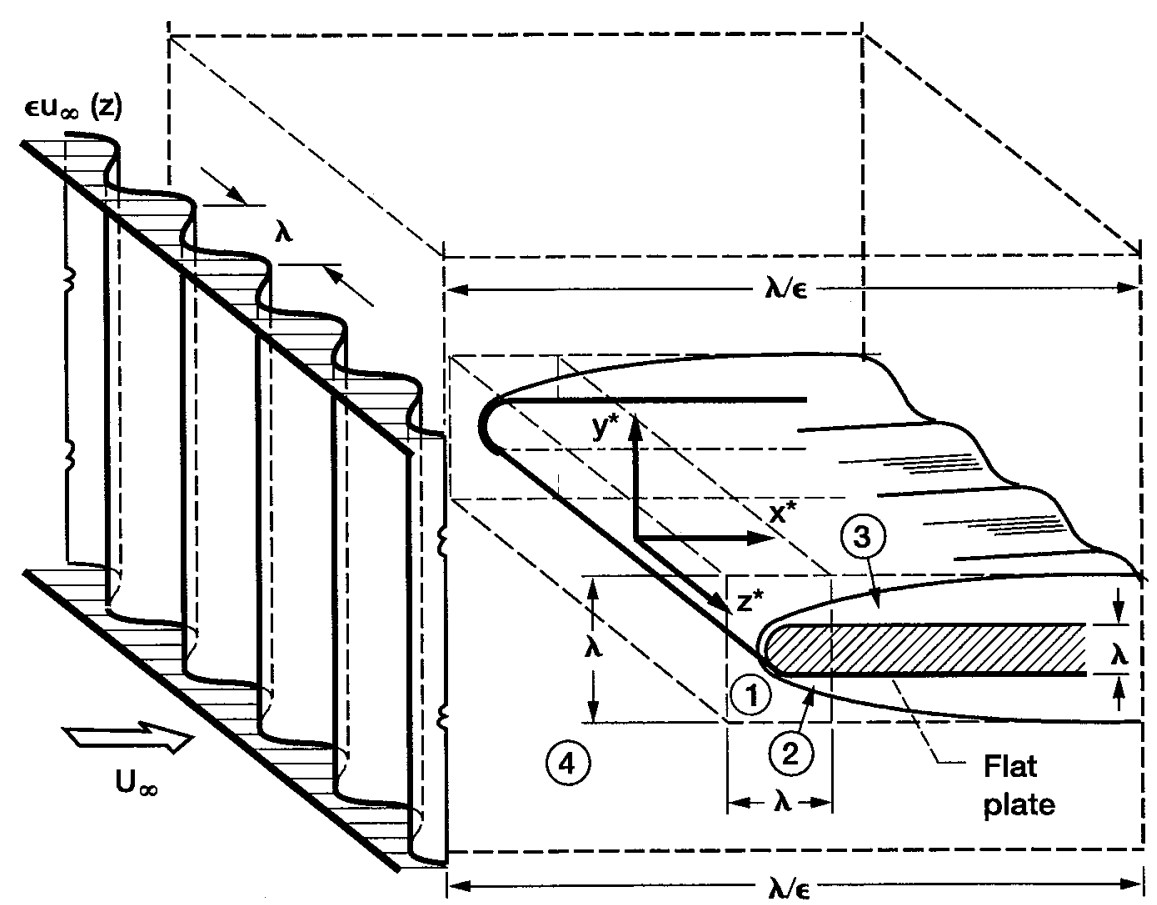

Figure 5.-Overall flow configuration showing various asymptotic regions considered in the analysis. 


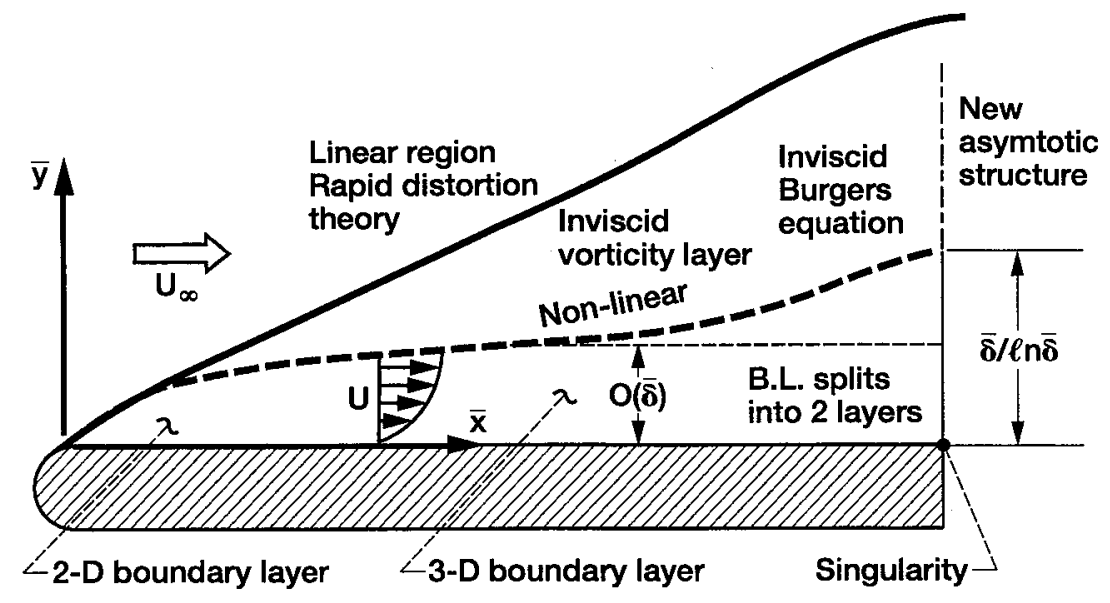

Figure 6.-Asymptotic flow structure in boundary layer in high disturbance Reynolds-number limit.

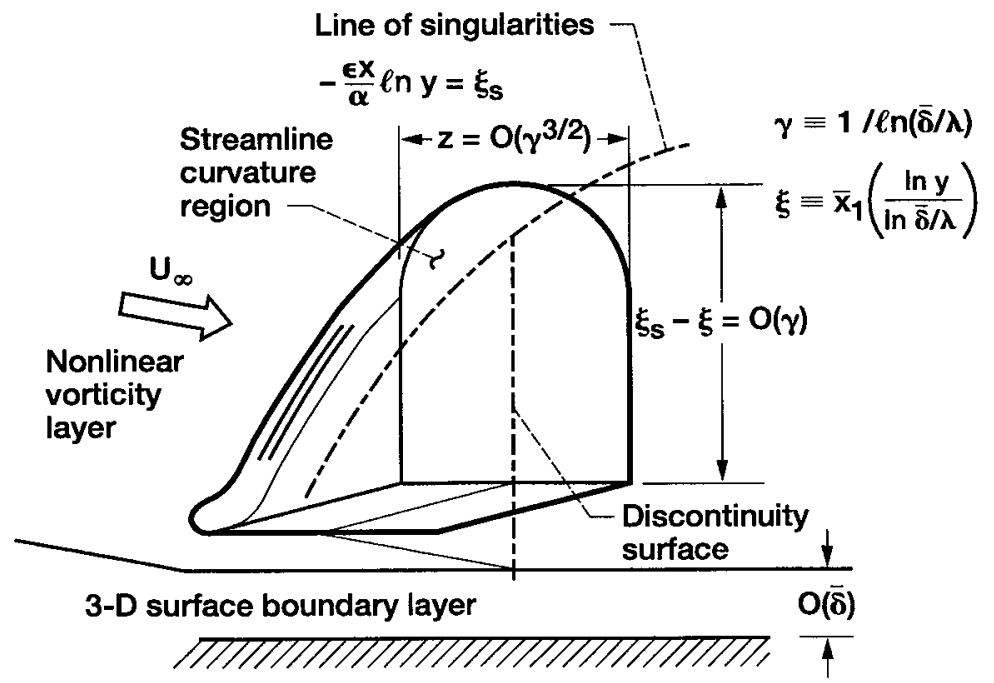

Figure 7.- Singularity structure of the nonlinear inviscid flow from Goldstein, Leib, and Cowley (1992). 

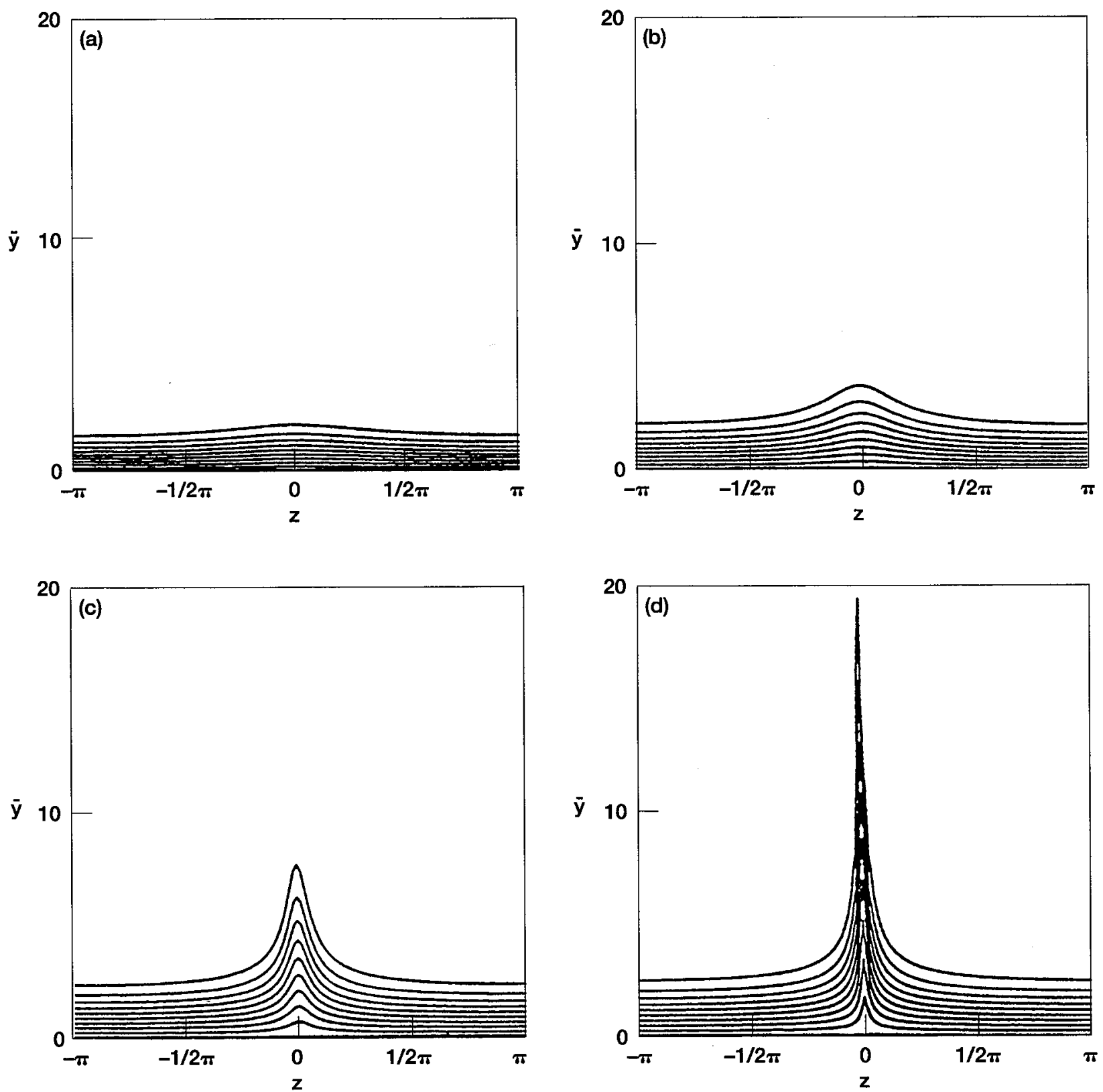

Figure 8.-Streamwise velocity contours in crossflow plane for $\bar{x}_{1}=$ (a) 0.25 , (b) 0.50 , (c) 0.75 , (d) 0.90 . The levels shown are from top to bottom; $U=0.9,0.8,0.7,0.6,0.5,0.4,0.3,0.2,0.1$ where $U$ is the magnitude of the streamwise velocity. 


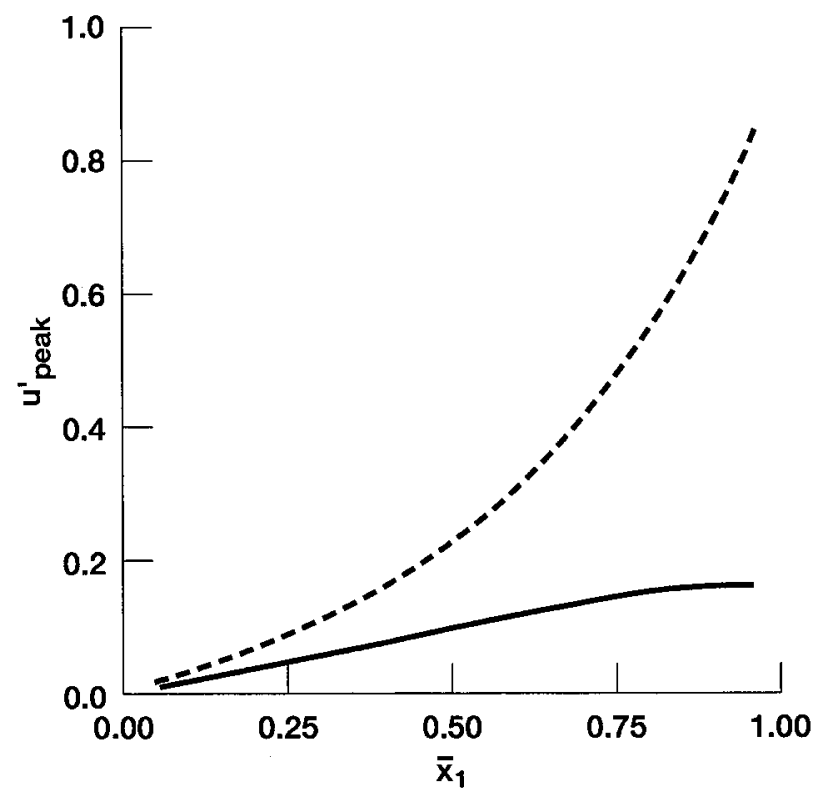

Figure 9.-Comparison of RMS and maximum velocity fluctuations in boundary layer as a function of the scaled streamwise coordinate $\bar{x}_{1}$. Solid line; peak value of $u^{\prime}\left(\bar{x}_{1}, \eta\right)$, dashed line; peak value of $\left|U\left(\bar{x}_{1}, \bar{y}, 0\right)-F_{B}^{\prime}(\eta)\right|$.

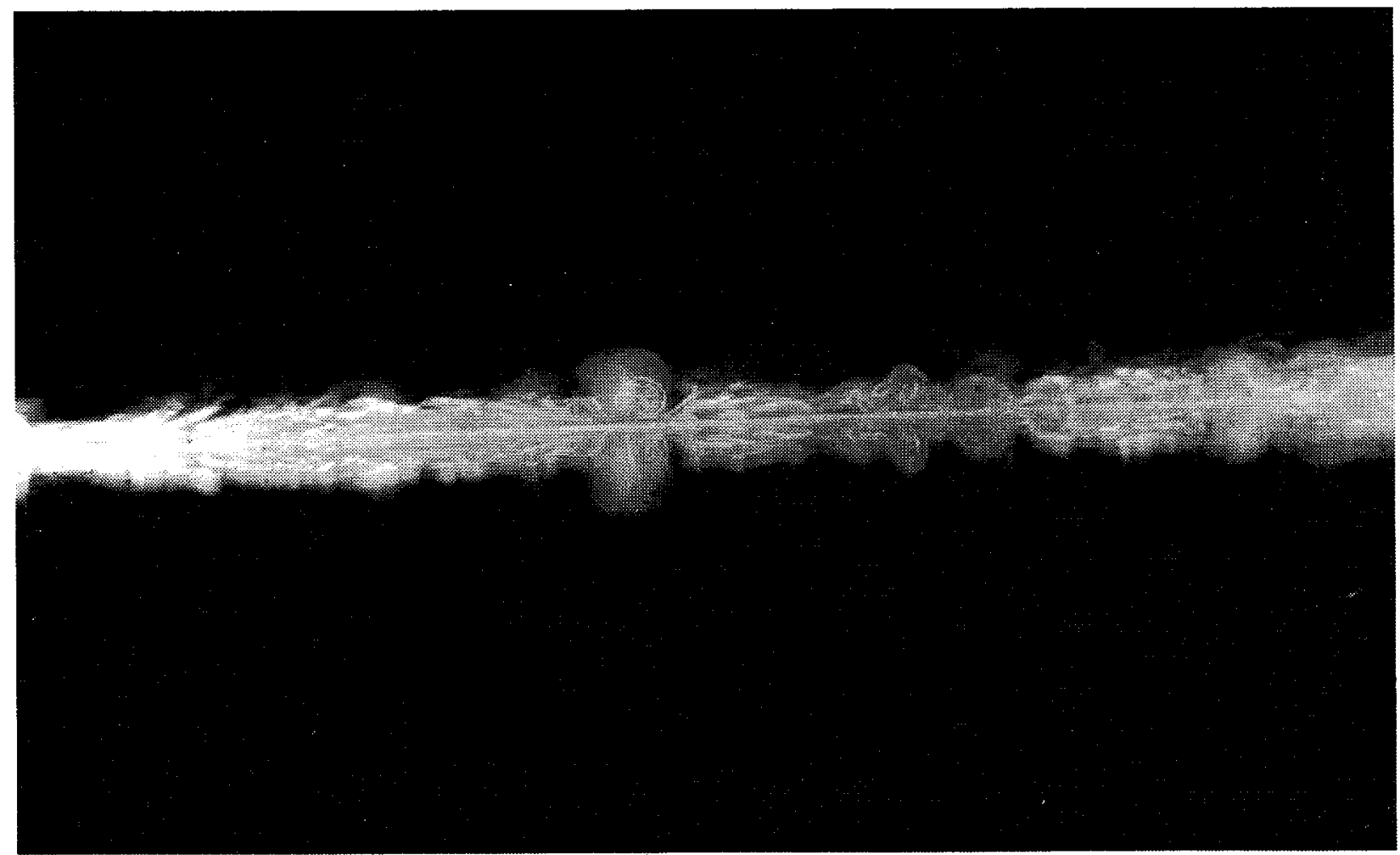

Figure 10.-Flow visualization of boundary layer beneath a turbulent stream (from Kendall, 1985). 


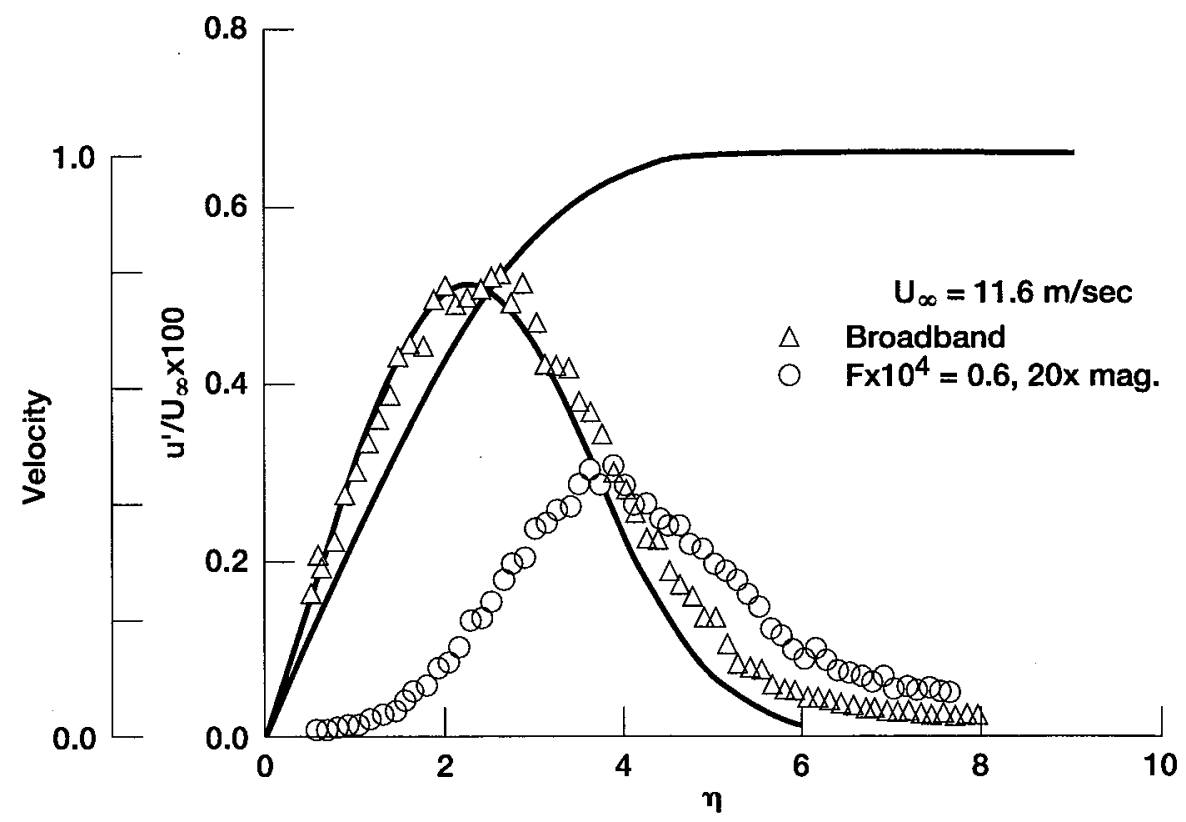

Figure 11.-Streamwise velocity fluctuations in boundary layer as measured by Kendall (1985). Turbulence is generated by a $6 \mathrm{~cm}$ grid $\sqrt{U_{\infty} X^{\star} / \nu}=717$.

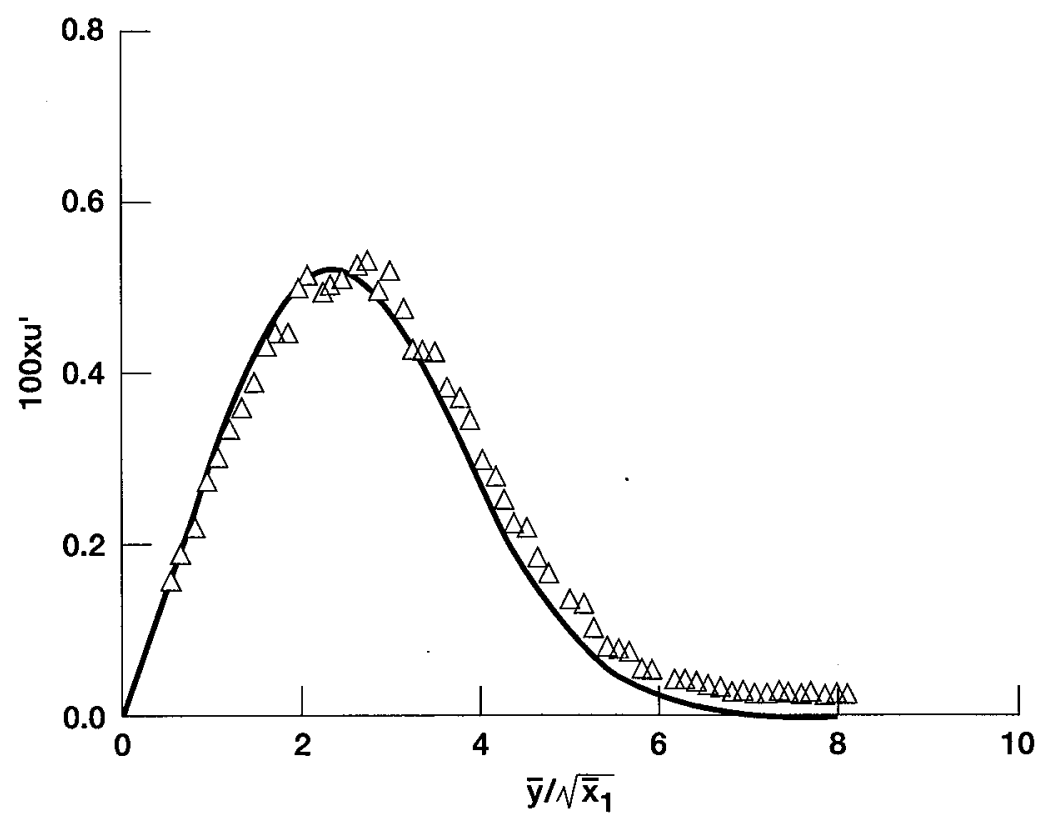

Figure 12.-Comparison of RMS velocity profile (18) at $\bar{x}_{1}=0.27$ with experimental results of Kendall (1985). 

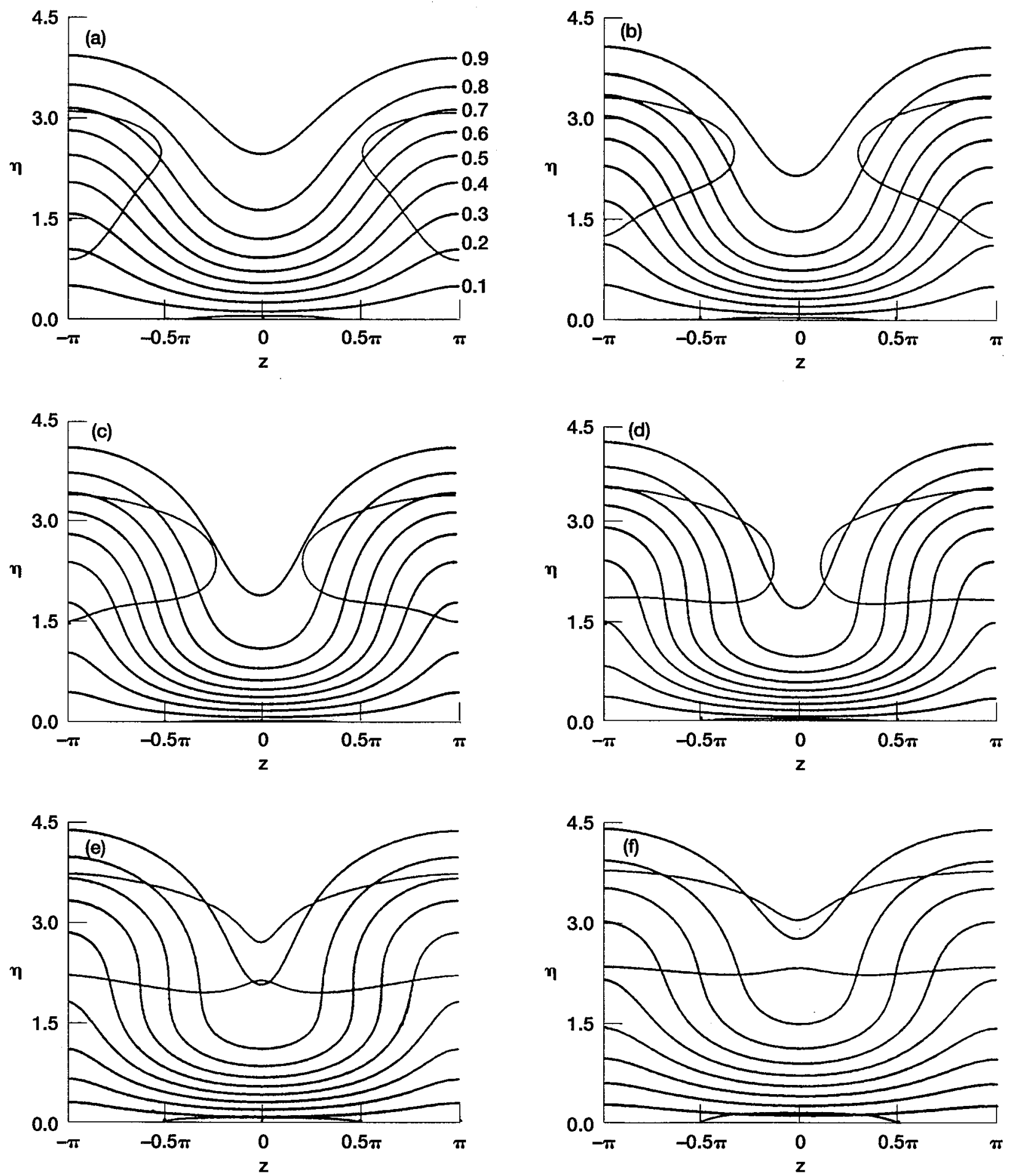

Figure 13. Streamwise velocity contours in the crossflow plane. Thick lines; $U$ contours, thin lines; inflexion points. (a) $\xi=1.1$. (b) $\xi=1.15$. (c) $\xi=1.2$. (d) $\xi=1.3$. (e) $\xi=1.5$. (f) $\xi=1.8$. $\xi=\epsilon X / \sigma+1$. 
Public reporting burden for this collection of information is estimated to average 1 hour per response, including the time for reviewing instructions, searching existing data sources, gathering and maintaining the data needed, and completing and reviewing the collection of information. Send comments regarding this burden estimate or any other aspect of this Davis Highway, Suite 1204, Artington, VA 22202-4302, and to the Office of Management and Budget, Paperwork Reduction Project (0704-0188), Washington, DC 20503.

\begin{tabular}{|l|l|l|}
\hline 1. AGENCY USE ONLY (Leave blank) & 2. REPORT DATE & 3. REPORT TYPE AND DATES COVERED
\end{tabular}

\begin{tabular}{|l|r|r|} 
1. AGENCYUSE ONLY (Leave blank) 1998 & Technical Memorandum \\
\hline
\end{tabular}

\section{TITLE AND SUBTITLE}

On the Environmental Realizability of Algebraically Growing Disturbances and Their Relation to Klebanoff Modes

\section{AUTHOR(S)}

Marvin E. Goldstein and David W. Wundrow

\section{PERFORMING ORGANIZATION NAME(S) AND ADDRESS(ES)}

National Aeronautics and Space Administration

Lewis Research Center

Cleveland, Ohio 44135-3191

9. SPONSORING/MONITORING AGENCY NAME(S) AND ADDRESS(ES)

National Aeronautics and Space Administration

Washington, DC 20546-0001

\section{FUNDING NUMBERS}

WU-537-05-21-00

8. PERForming ORGANIZATION REPORT NUMBER

E-10995

10. SPONSORING/MONITORING AGENCY REPORT NUMBER

NASA TM-1998-206298

\section{SUPPLEMENTARY NOTES}

Prepared for the International Symposium Theoretical and Computational Fluid Dynamics sponsored by the Florida State University, Tallahassee, Florida, November 6-8, 1996. Marvin E. Goldstein, NASA Lewis Research Center, and David W. Wundrow, NYMA, Inc., 2001 Aerospace Parkway, Brook Park, Ohio 44142 (work funded by NASA Contract NAS3-27186). Responsible person, Marvin E. Goldstein, organization code 0100, (216) 433-5825.

12a. DISTRIBUTION/AVAILABILITY STATEMENT

Unclassified - Unlimited

Subject Category: 34

Distribution: Nonstandard

This publication is available from the NASA Center for AeroSpace Information, (301) 621-0390.

13. ABSTRACT (Maximum 200 words)

A theoretical explanation of some experimentally observed phenomena associated with the so-called Klebanoff modes is obtained by analyzing the flow over a finite thickness flat plate resulting from a small-amplitude distortion imposed on the upstream mean flow. The analysis shows (among other things) how the stretching of the vortex lines around the plate leads to streamwise vorticity at the plate surface, which then produces a streamwise velocity perturbation within the boundary layer that can be related to the experimentally observed Klebanoff mode. The complete evolution of this flow must be found by solving the boundary-region equations of Kemp (1951) and Davis and Rubin (1980), but a limiting analytical solution can also be obtained. Since the initial growth of the boundary-layer disturbance is nearly algebraic, our results demonstrate how the algebraically growing disturbances promoted by Landahl and others can be generated by a realistic external-disturbance environment. The relationship between these results and various bypass transition mechanisms is discussed.

\section{SUBJECT TERMS}

Transition; Boundary layer instability; Turbulence

\begin{tabular}{|c|c|}
\hline $\begin{array}{c}\text { 17. SECURITY CLASSIFICATION } \\
\text { OF REPORT } \\
\text { Unclassified }\end{array}$ & $\begin{array}{c}\text { 18. SECURITY CLASSIFICATION } \\
\text { OF THIS PAGE } \\
\text { Unclassified }\end{array}$ \\
\hline
\end{tabular}

19. SECURITY CLASSIFICATION OF ABSTRACT Unclassified
15. NUMBER OF PAGES 24

16. PRICE CODE

$\mathrm{A03}$

20. LIMITATION OF ABSTRACT 
